\title{
Analysis of Service Efficiency of Traditional Chinese Medicine (TCM) Hospitals in China Based on Data Envelopment Analysis (DEA)
}

\author{
ZHAO Jian-yao ${ }^{1}$, SHUAI Li-na ${ }^{1}$, GUO Fang ${ }^{2}$, CHEN Li ${ }^{1, *}$ \\ ${ }^{1}$ School of Management, Hubei University of Chinese Medicine, Wuhan 430065, China \\ ${ }^{2}$ Shandong Bausch \& Lomb and Freda Pharmaceutical Co., Ltd., Jinan 250000, China
}

\begin{abstract}
In order to optimize the allocation of health resources and promote the development of traditional Chinese medicine (TCM), this research employs the China Health Statistics Yearbook 2018 to collect relevant data on TCM hospitals in 2017, and the DEA method is used to construct a BCC model for the evaluation of service efficiency of these hospitals in 30 provinces (including autonomous regions and municipalities directly under the central government) across the country. It is found that TCM hospitals in non-effective DEA provinces are not operating efficiently, with excessive investment in institutions, beds, and health staff, and insufficient output of visits, bed utilization, and the average length of stay. Given this, attention to promoting the service capacity and efficiency of TCM hospitals should be paid on the following aspects: scientific allocation of health resource, strengthened external policy control, improved internal management of hospitals, optimized characteristic services and establishment of scientific evaluation system.
\end{abstract}

\section{Introduction}

Since the 18th National Congress of the CPC, the Party Central Committee has attached great importance to the cause of TCM and has dedicated to improving the capacity of TCM services. For TCM hospitals being important vehicles for the provision of TCM services in China, its service efficiency provided is directly related to the development of TCM. Based on the statistics of TCM hospitals in 30 provinces (including autonomous regions and municipalities directly under the central government) in 2017, the Data Envelopment Analysis (DEA) is used to analyze the service efficiency of TCM hospitals in each province, and provide decision references for optimizing the allocation of health resources and improving the service efficiency.

\section{Source and Research Methodology}

\subsection{Source}

The research data, deriving from the 2018 China Statistical Yearbook of Health, has excerpted the relevant statistics of TCM hospitals in 30 provinces in 2017, including autonomous regions and municipalities directly under the central government. Tibet Autonomous Region is not included in this study for its lack of TCM hospital.

\subsection{Research Methodology}

DEA was initially created by Charnes and Cooper in 1978. It is a systematic analysis method based on the concept of relative efficiency and mathematical programming theory to evaluate the relative effectiveness of multi-input and multi-output decision-making units ${ }^{[1]}$. This study uses DEAP2.1 to build the BCC model so as to evaluate the relative effectiveness of pure technical efficiency of TCM hospitals in 30 provinces (including autonomous regions and municipalities directly under the central government) in China.

\section{Results and Analysis}

\subsection{Establishment of Research Indicators}

When using DEA to evaluate the technical efficiency of TCM hospital's health resource input and output projects, the results are directly determined by whether the representative indicator selection is reasonable or not. Through literature studies and summaries, the input indicators are identified as the number of institutions, beds and the number of health staff in TCM hospitals, and the output indicators are the number of hospital visits, discharges, bed utilization rate and the average length of stay. The specific details of each indicator are shown in Table 1. 
Table 1. Description statistics of input and output indicators based on DEA.

\begin{tabular}{|c|c|c|c|c|}
\hline & Maximum & Minimum & Mean & Standard deviation \\
\hline Number of institutions & 263 & 13 & 122.97 & 66.36 \\
\hline Number of beds & 63,144 & 2,445 & $27,273.87$ & $17,096.36$ \\
\hline Number of health staff & 62,465 & 2,238 & $26,566.8$ & $16,966.22$ \\
\hline Medical visits (thousand) & 52,772 & 1,405 & $17,616.4$ & $13,141.6$ \\
\hline Number of discharges & $1,857,997$ & 82,466 & $827,287.27$ & $552,077.58$ \\
\hline Bed utilization rate (\%) & 97.3 & 67.5 & 83.59 & 16.92 \\
\hline Average length of stay (day) & 12.3 & 8.2 & 9.7 & 0.9 \\
\hline
\end{tabular}

\subsection{Results of Service Efficiency of TCM Hospitals Based on DEA}

The health resource allocation efficiency of TCM hospitals in 30 provinces (including autonomous regions and municipalities directly under the central government) in 2017 are found out through calculating the selected input indicators and output indicators within the BCC model constructed with the use of DEAP2.1. And the specific results are shown in Table 2.

Table 2. Effectiveness evaluation of TCM hospitals in 30 provinces in 2017.

\begin{tabular}{|c|c|c|c|c|}
\hline Province & Technical efficiency & Pure technical efficiency & Scale efficiency & Returns to scale \\
\hline Beijing & 0.760 & 1.000 & 0.760 & Decreasing \\
\hline Tianjin & 0.711 & 1.000 & 0.711 & Decreasing \\
\hline Hebei & 0.830 & 0.865 & 0.960 & Decreasing \\
\hline Shanxi & 0.576 & 0.864 & 0.667 & Decreasing \\
\hline Inner Mongolia & 0.661 & 0.845 & 0.783 & Decreasing \\
\hline Liaoning & 0.695 & 0.986 & 0.705 & Decreasing \\
\hline Jilin & 0.634 & 0.815 & 0.778 & Decreasing \\
\hline Heilongjiang & 0.669 & 0.814 & 0.822 & Decreasing \\
\hline Shanghai & 1.000 & 1.000 & 1.000 & Unchanged \\
\hline Jiangsu & 1.000 & 1.000 & 1.000 & Unchanged \\
\hline Zhejiang & 0.784 & 1.000 & 0.784 & Decreasing \\
\hline Anhui & 1.000 & 1.000 & 1.000 & Unchanged \\
\hline Fujian & 0.770 & 0.788 & 0.977 & Decreasing \\
\hline Jiangxi & 0.905 & 0.931 & 0.971 & Decreasing \\
\hline Shandong & 0.835 & 0.928 & 0.899 & Decreasing \\
\hline Henan & 0.796 & 1.000 & 0.796 & Decreasing \\
\hline Hubei & 0.996 & 1.000 & 0.996 & Decreasing \\
\hline Hunan & 1.000 & 1.000 & 1.000 & Unchanged \\
\hline Guangdong & 0.870 & 1.000 & 0.870 & Decreasing \\
\hline Guangxi & 0.961 & 1.000 & 0.961 & Decreasing \\
\hline Hainan & 0.799 & 0.804 & 0.994 & Increasing \\
\hline Chongqing & 0.935 & 0.979 & 0.955 & Decreasing \\
\hline Sichuan & 0.952 & 1.000 & 0.952 & Decreasing \\
\hline
\end{tabular}




\begin{tabular}{|c|c|c|c|c|}
\hline Guizhou & 1.000 & 1.000 & 1.000 & Unchanged \\
\hline Yunnan & 0.944 & 0.978 & 0.966 & Decreasing \\
\hline Shaanxi & 0.848 & 0.927 & 0.915 & Decreasing \\
\hline Gansu & 1.000 & 1.000 & 1.000 & Unchanged \\
\hline Qinghai & 1.000 & 1.000 & 1.000 & Decreasing \\
\hline Ningxia & 0.853 & 1.000 & 0.853 & Decreasing \\
\hline Xinjiang & 0.996 & 1.000 & 0.996 & \\
\hline
\end{tabular}

\subsubsection{Technical efficiency Analysis}

The average technical efficiency of TCM hospitals in 30 provinces (including autonomous regions and municipalities directly under the central government) in China is 0.859 , and varies greatly from region to region. Among them, Shanghai, Jiangsu, Anhui, Hunan, Guizhou, Gansu, and Qinghai Provinces achieved effective DEA, accounting for $23.33 \%$ of the total. This shows that the overall technology and management level of TCM hospitals in these 7 provinces (including autonomous regions and municipalities directly under the central government) is high, and health resources are fully invested and utilized. The maximum output is basically achieved, and input indicators and output indicators achieve the highest level of medical service efficiency ${ }^{[2]}$. When based on geographical location, the effective ratio of DEA in the eastern, central, and western regions is $16.67 \%, 22.22 \%$, and $33.33 \%$ respectively. The western region is slightly higher than the other two regions, with a technical efficiency of $53.33 \%$ of the decision-making units being less than 0.9. Most TCM hospitals in these areas have problems with poor operating efficiency, redundant inputs or insufficient output, and attention should be paid to adjusting the input-output structure and improving management to promote TCM hospitals' service efficiency and development ${ }^{[3]}$.

\subsubsection{Pure technical efficiency Analysis}

Pure technical efficiency refers to the maximum output that the selected decision-making unit can obtain under the current input level ${ }^{[4]}$. The average pure technical efficiency of TCM hospitals in 30 provinces (including autonomous regions and municipalities directly under the central government) is 0.951 , and 17 of them have achieved effective pure technical efficiency, accounting for $56.67 \%$. The proportions of effective pure technical efficiency in eastern, central and western regions are $58.33 \%, 44.44 \%$ and $66.67 \%$ respectively. In addition to the 7 provinces with effective DEA, 10 provinces including Beijing, Tianjin, Zhejiang, Henan as well as other provinces (including autonomous regions and municipalities directly under the central government) have also reached pure technical efficiency. Though these regions have realized the full use of health resources, the invalid scale and the decreasing returns to scale show that the marginal income is decreasing with the expansion of the scale.

\subsubsection{Scale Efficiency Analysis}

The average scale efficiency of TCM hospitals in 30 provinces (including autonomous regions and municipalities directly under the central government) is 0.902. Except for the 7 provinces (including autonomous regions and municipalities directly under the central government) that have achieved effective DEA, none of them have achieved scale efficiency. Among them, Hainan Province enjoys increasing returns to scale, that is, output efficiency increases with scale expansion, and the output factors grow higher than that of production ${ }^{[5]}$. The other 22 provinces (including autonomous regions and municipalities directly under the central government) have decreasing returns to scale. In these regions attention should be paid to policy regulation, management improvement and other ways to enhance scale efficiency.

\subsection{Analysis of Slack Variables in Non-effective DEA Provinces}

Through the analysis of slack variables in non-effective DEA provinces (including autonomous regions and municipalities directly under the central government), we can fully realize the insufficiency or surplus of input and output. There are 23 non-effective DEA provinces (including autonomous regions, municipalities directly under the central government), of which 10 are technically effective, with zero slack variable. And the pure technical efficiency of the rest regions is less than 1 , which means the current input level does not achieve the maximum output. The slack variables are shown in Table 3. 
Table 3. Slack variables of input and output of TCM hospitals in non-effective DEA provinces.

\begin{tabular}{|c|c|c|c|c|c|c|c|}
\hline & $\begin{array}{c}\text { Number of } \\
\text { institutions }\end{array}$ & $\begin{array}{c}\text { Number of } \\
\text { beds }\end{array}$ & $\begin{array}{c}\text { Number of } \\
\text { health staff }\end{array}$ & Medical visits & $\begin{array}{c}\text { Number of } \\
\text { discharges }\end{array}$ & $\begin{array}{c}\text { Bed utilization } \\
\text { rate }\end{array}$ & $\begin{array}{c}\text { Average length } \\
\text { of stay (day) }\end{array}$ \\
\hline Hebei & 78.65 & 0.00 & 0.00 & 0.00 & 0 & 14.47 & 0.39 \\
\hline Shanxi & 115.61 & $1,961.27$ & 0.00 & 693.20 & 0 & 10.81 & 0.00 \\
\hline Inner Mongolia & 42.12 & 0.00 & 0.00 & 402.54 & 0 & 10.31 & 0.00 \\
\hline Liaoning & 47.22 & $5,339.67$ & 0.00 & 827.39 & 0 & 5.25 & 0.00 \\
\hline Jilin & 9.14 & 0.00 & 0.00 & 405.37 & 0 & 7.58 & 0.00 \\
\hline Heilongjiang & 32.49 & 179.65 & 0.00 & 528.23 & 0 & 8.64 & 0.00 \\
\hline Fujian & 15.05 & 0.00 & 200.80 & 4.95 & 0 & 19.26 & 0.00 \\
\hline Jiangxi & 0.00 & 0.00 & 0.00 & 73.58 & 0 & 8.04 & 0.00 \\
\hline Shandong & 23.96 & 0.00 & $9,392.33$ & 19.91 & 0 & 12.87 & 0.88 \\
\hline Hainan & 0.42 & 0.00 & 249.68 & 150.68 & 0 & 15.36 & 0.37 \\
\hline Chongqing & 0.00 & 0.00 & 0.00 & 0.00 & 0 & 1.78 & 0.00 \\
\hline Yunnan & 23.49 & 0.00 & 0.00 & 0.00 & 0 & 5.60 & 0.12 \\
\hline Shaanxi & 47.06 & 0.00 & $3,644.20$ & 318.71 & 0 & 2.61 & 0.00 \\
\hline
\end{tabular}

Through analysis, it is found that if all TCM hospitals in 30 provinces (including autonomous regions and municipalities directly under the central government) achieve effective DEA, a total of 435 institutions will be streamlined, 7,481 beds will be saved, 13,487 health staff will be reduced, 34,245,500 visits will increase, the bed utilization rate will increase by an average of $4.09 \%$, and the average length of stay will increase by 0.059 days.

In terms of investment indicators, the most redundant are the 115.61 excessive institutions in Shanxi Province, 5,339.67 beds in Liaoning Province, and 9,392.33 health staff in Shandong Province. The redundancy rates are $54.28 \%, 19.74 \%$, and $15.04 \%$ respectively. In terms of output indicators, with the full utilization of input factors, the biggest improvement in output is the increase of 8.2739 million visits in Liaoning Province, the increase of hospital bed utilization rate in Fujian Province by $19.26 \%$, and the increase of the average length of stay in Shandong Province by 0.88 days, with the growth rates of $77.78 \%, 25.14 \%$ and $9.36 \%$ respectively.

\section{Discussion and Suggestions}

\subsection{The service efficiency of TCM hospitals in various regions of China being generally low and varying greatly}

In 2017, the service efficiency of TCM hospitals in all provinces (autonomous regions and municipalities directly under the central government) across the country was generally low with an average technical efficiency of 0.859 . And $53.33 \%$ of the provinces had a technical efficiency of less than 0.9 , while the lowest value was only 0.576 . There are great differences among provinces. Social and economic development as well as constraints of the TCM development in various regions lead to a large gap in service efficiency and uneven developments of TCM hospitals in the eastern, central and western regions. Provinces (autonomous regions and municipalities directly under the central government) with effective DEA in the eastern, central and western regions account for $16.67 \%, 22.22 \%$ and $33.33 \%$ respectively. The western region is slightly better than the two others. Thus, it can be concluded that improving service efficiency is a common task for most TCM hospitals.

\subsection{To improve the pure technical efficiency of TCM hospitals by allocating health resources scientifically}

In $2017,56.67 \%$ of the provinces (including autonomous regions and municipalities directly under the central government) achieved effective pure technical efficiency, with an average pure technical efficiency of 0.951 . However, there were still TCM hospitals in some areas that have redundant inputs and insufficient outputs, resulting in a waste of health resources. In view of this, in the process of health resource allocation, we shall reasonably plan the geographical distribution of health services, fully consider the differences of local population development trend, economic level, demand for TCM health services and other conditions ${ }^{[6]}$, and focus on increasing investment in TCM hospitals in remote, densely populated and vulnerable areas with relatively scarce medical and health resources, so as to improve the TCM service system ${ }^{[7]}$, the equity of health resource allocation and the service efficiency of TCM hospital.

\subsection{To strengthen policy regulation and improve the scale efficiency of TCM hospitals}

In 2017, the average scale efficiency of TCM hospitals in all provinces (including autonomous regions and 
municipalities under the central government) in China was 0.902 , and 23 provinces (including autonomous regions and municipalities under the central government) did not achieve scale efficiency. Among the 23 provinces, $95.5 \%$ was diminishing in scale efficiency, which means with the expansion of scale, the output efficiency decreases and the production factors grow higher than the output. TCM hospitals should rationally manage the hospital scale, strengthen policy regulation, change the simple development method of blindly expanding the scale, purchasing expensive medical equipment, and expanding the building area, so as to improve the rational allocation of existing health resources and realize full use of them. Besides, a sound external environment should be created for the development of TCM hospitals by formulating public policy. For example, the Shanghai municipal government has launched the practical technology promotion project of TCM and built the "national suitable technology promotion base for common and frequentlyoccurring diseases at grass-roots level ", which has increased the outpatient income and medical visits of TCM hospitals in these areas, made full use of health resource investment, and greatly improved the service efficiency of TCM hospitals in Shanghai ${ }^{[8]}$.

\subsection{To improve the service efficiency of TCM hospitals by advancing management and building a scientific evaluation system}

In order to tackle the overall input redundancy, insufficient output and low operation efficiency, TCM hospitals should make a clear development strategy for the hospital, learn advanced management concepts to refine its management, and optimize workflow to reduce loss and errors. What's more, hospitals should prioritize its efficiency improvement, make full use of existing resources, and improve service efficiency to achieve Pareto optimality.

Additionally, in order to improve the service efficiency, TCM hospitals should strictly follow the relevant national regulations to carry out comprehensive performance appraisals for the timely grasp of its operation condition and scientifical evaluation of the service efficiency. When analyzing and evaluating the service efficiency of TCM hospitals, it is necessary to establish a medical information management system in line with the characteristics of TCM diagnosis and treatment in combination with modern information technology and appropriately add the characteristic indicators of TCM, and input and output indicators related to the government and patients as the basis for the examination and evaluation of TCM hospitals $^{[9]}$.

\subsection{To enhance the key competitiveness of TCM hospitals by vigorously developing special service projects of TCM}

Some studies have shown that the unsatisfactory service efficiency of TCM hospitals may be related to the following factors in recent years: "Westernization of TCM", the lack of TCM talents, distinctive TCM services overshadowed by Western medicine, diminished advantages of TCM, and the relatively slow development of TCM technology ${ }^{[10]}$. Therefore, we shall promote the development of TCM departments in the hospital and improve clinical quality and service efficiency by clarifying the service positioning of TCM hospitals, strengthening its own internal development and construction, utilizing unique local advantages, and vigorously developing characteristic service projects of TCM. Moreover, we should focus on building disease specialty that can reflect the obvious curative effect of TCM on the basis of the original construction of key disciplines of TCM and play a leading role.

\section{References}

1. Coelli T.J, Rao Dsp, O’Donnell CJ, et al. An Introduction to Efficiency and Productivity Measurement [M]. New York: Springer, 2005:67-69.

2. Wang Ying, Huang Minyuan, Wei Jianhao, et al. Research on the Efficiency and Prediction of Medical Service in Hunan Provincial Hospital of TCM [J]. Chinese Journal of Information on Traditional Chinese Medicine, 2018, 25(10): 4-8.

3. Lv Yijuan, Tao Wanju. Basic Situation and Service Efficiency Evaluation of Traditional Chinese Medicine Hospitals in 2015 [J]. Modern Business Trade Industry, 2018, 39 (02): 82-84.

4. Shen Junlong, Tang Shaoliang, Bian Qijuan, et al. Research on Scientific and Technological Innovation Efficiency of Traditional Chinese Medicine Hospitals in Jiangsu Province Based on DEA [J]. Science and Technology Management Research, 2012,32 (17): 42-48.

5. Yuan Jia, Zhao Liying, Man Xiaowei, et al. Operational Efficiency Analysis of 72 Tertiary Traditional Chinese Medicine Hospitals in Western China based on DEA [J]. Medicine and Society, 2015, 28(04): 47-49+60.

6. Zhang Xinnan, Yang Yi, Chen Zhaorong, etc. Evaluation on the Efficiency of Health Resource Allocation of Traditional Chinese Medicine Hospitals across China Based on DEA [J]. Chinese Hospital Management, 2017, 37(11): 30-33.

7. Zhou Xiaojian, Jiang Guanxu. Evaluation and Analysis of Health Resource Allocation Efficiency Based on DEA [J]. Modern Preventive Medicine, 2010, 37 (20): 3873-3875.

8. Shi Yun, Shi Xiaolin, Jia Yang, et al. Current Situation and Policy Suggestions on the Use of Appropriate Technologies of Traditional Chinese Medicine in Shanghai Communities [J]. Journal of Traditional Chinese Medicine, 2019, 27 (18): 200202.

9. Lu Wenliang, Zeng Cheng, Chen Lili, et al. Evaluation of Hospital Operation Efficiency Based on DEA [J]. Chinese Digital Medicine, 2017, 12(11): 92-94.

10. Chen Fang, Xiang Yuanwei, Jiang Jianhua, et al. 
Analysis of Efficiency and Influencing Factors of Guangdong Provincial Hospital of TCM [J]. Chinese Health Service Management, 2018, 35(10): 744-747. 